\title{
Dielectric versus topographic contrast in near-field microscopy
}

\author{
Olivier J. F. Martin \\ Laboratory for Field Theory and Microwave Electronics, Swiss Federal Institute of Technology, ETH-Zentrum, \\ 8092 Zurich, Switzerland \\ Christian Girard \\ Laboratoire de Physique Moléculaire, Unité de Recherche Associée au Centre de la Recherche Scientifique 772, \\ Université de Franche-Comté, 25030 Besançon, France
}

\begin{abstract}
Alain Dereux
Equipe Submicronique, Unité de Recherche Associée au Centre de la Recherche Scientifique 1796, Laboratoire de Physique, Université de Bourgogne, BP 138, 21004 Dijon Cedex, France
\end{abstract}

Received October 30, 1995; accepted January 22, 1996

\begin{abstract}
Using a fully vectorial three-dimensional numerical approach (generalized field propagator, based on Green's tensor technique), we investigate the near-field images produced by subwavelength objects buried in a dielectric surface. We study the influence of the object index, size, and depth on the near field. We emphasize the similarity between the near field spawned by an object buried in the surface (dielectric contrast) and that spawned by a protrusion on the surface (topographic contrast). We show that a buried object with a negative dielectric contrast (i.e., with a smaller index than its surrounding medium) produces a near-field image that is reversed from that of an object with a positive contrast. (C) 1996 Optical Society of America.
\end{abstract}

\section{INTRODUCTION}

With a resolution far beyond the diffraction limit, scanning near-field optical microscopy (SNOM) is becoming an extremely powerful technique for the analysis of surface structures at the mesoscopic scale. ${ }^{1}$ [SNOM as used in this paper also covers near-field scanning optical microscopy (NSOM), photon scanning tunneling microscopy (PSTM), and scanning tunneling optical microscopy (STOM).]

The imaging properties of the topographic variations of a surface (protrusions, surface roughness) have been investigated from a theoretical point of view with different numerical methods, and the relationship between a topographic object and its near-field image is understood to a certain extent. $^{2-9}$ Recently, procedures have also been proposed for solving the important inverse problem, i.e., the reconstruction of the topographic profile from the near-field data. ${ }^{10-13}$ On the other hand, to the best of our knowledge, pure dielectric contrast has been investigated only in two-dimensional systems or by means of a perturbative approach, ${ }^{14-16}$ and no self-consistent calculations have yet been presented on pure dielectric contrast for three-dimensional (3D) defects buried in a dielectric surface. In this paper we address this problem and investigate the near field spawned by a perfectly flat surface displaying 3D index variations.

The physical system considered and the formalism used are described in Section 2. In Section 3 we study the influence on the near field of different physical param- eters (index contrast, size, and depth of the perturbation). These results are summarized in Section 4.

\section{MODEL}

Local index variations of a flat dielectric surface are commonly used in integrated optics to define buried waveguides. Such diffused waveguides represent key components for advanced photonic integrated circuits (PIC's). Depending on the substrate material used, they can be produced by different techniques: ion exchange, flame hydrolysis, or chemical vapor deposition. ${ }^{17,18}$ It is important to note that all these techniques produce extremely weak index variations in the surface, $\Delta n \leqslant 0.01{ }^{19}$

The analysis and monitoring of photonic integrated circuits indisputably provides an extremely promising application field for SNOM. ${ }^{20-22}$ We will therefore place the present study in this context and investigate SNOM's imaging properties of weak index variations, similar to those used for diffused waveguides in PIC's.

In this paper we consider a flat dielectric surface $(n=1.500)$ with localized $3 \mathrm{D}$ index variations $\Delta n<0.01$. The surface is illuminated from below by total reflection (Fig. 1). For such a glass-air interface, the total reflection angle is $42^{\circ}$; we take for our calculations an incident angle of $50^{\circ}$. We investigate two different incident polarizations: $s$ polarization, where the incident electric field is parallel to the glass-air interface, and $p$ polarization, where it is in the plane of incidence (Fig. 1). 
The electric near field spawned above the surface is computed with the generalized field propagator; this fully vectorial formalism, based on Green's tensor technique, allows simultaneous computation of the responses of a physical system to different incident fields. ${ }^{23}$ In this way, different polarizations and incident field directions can be investigated simultaneously. For all the results presented in this paper, we consider an illumination wavelength of $633 \mathrm{~nm}$ in vacuum and a $5 \times 5 \times 5 \mathrm{~nm}^{3}$ discretization mesh for the inhomogeneities of the system. ${ }^{24}$

\section{RESULTS}

To compare topographic and dielectric contrasts, let us first consider a pure topographic case, where a $20 \times 20 \times 10$ $\mathrm{nm}^{3}$ protrusion with the same index is placed on the surface (Fig. 1). In this figure we report the relative total electric field intensity in a plane parallel to the surface, located $5 \mathrm{~nm}$ above the protrusion. The field intensity $I$ is normalized to the intensity $I_{0}$ that would be measured without a protrusion.

For $p$ polarization [Fig. 1(a)] we observe a strong field confinement that reproduces the object shape. ${ }^{5}$ The fact that an object much smaller than the wavelength can spawn a confined field that perfectly reproduces its shape explains how SNOM achieves a resolution far beyond the diffraction limit. Indeed, such a confined field can be detected by a SNOM probing tip.

For $s$ polarization, the field intensity no longer reproduces the object, but strong field gradients appear along the object sides that are orthogonal to the incident field, whereas the object itself appears in reversed contrast: A depletion in the field intensity is observed above the object [Fig. 1(b)].

This difference of behavior between $s$ and $p$ polarizations, which is also observed experimentally, ${ }^{25}$ is easily understood if one recalls the fact that a small volume of matter, such as the protrusion in Fig. 1, generates a depolarization field $\mathbf{E}^{d}$ when it is submitted to an external field $\mathbf{E}^{0}$. This depolarization field is such that the total field $\mathbf{E}=\mathbf{E}^{0}+\mathbf{E}^{d}$ fulfills the boundary conditions required by Maxwell equations. ${ }^{26}$ For $s$ polarization the discontinuity of the total field along the protrusion sides orthogonal to the incident field imposes strong variations of the depolarization field along these sides [Fig. 1(b)]. On the other hand, the incident field already satisfies (a) p-polarization

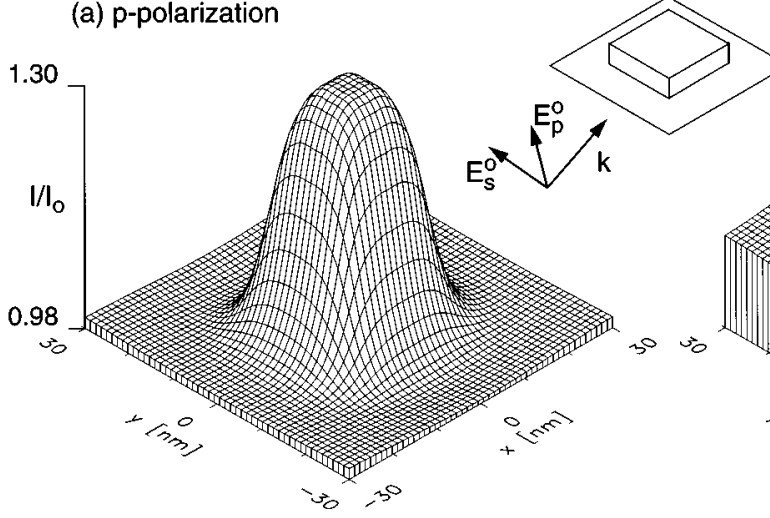

(b) s-polarization

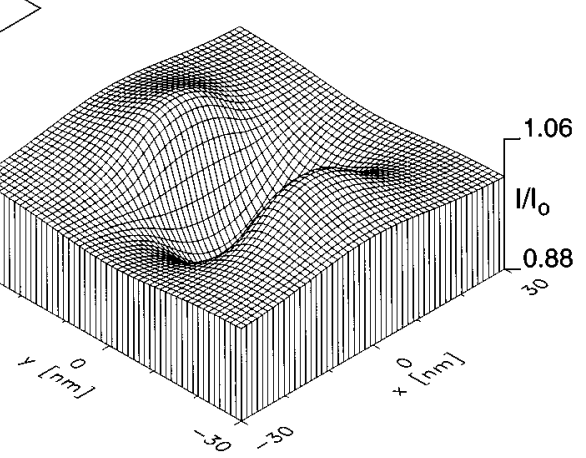

Fig. 1. Relative total field intensity $I / I_{0}$, above a dielectric surface $(n=1.500)$ with a $20 \times 20 \times 10 \mathrm{~nm}^{3}$ protrusion of same index. The field intensity $I$ is computed $5 \mathrm{~nm}$ above the protrusion (i.e., $15 \mathrm{~nm}$ above the surface) and normalized with the value $I_{0}$ that would be obtained without a protrusion. The surface is illuminated by total reflection with a wave propagating in the $k$ direction. Two different incident polarizations are investigated: (a) $p$ polarization (incident electric field $E_{p}^{0}$ ) and (b) $s$ polarization (incident electric field $\left.E_{s}^{0}\right)$.

(a) p-polarization

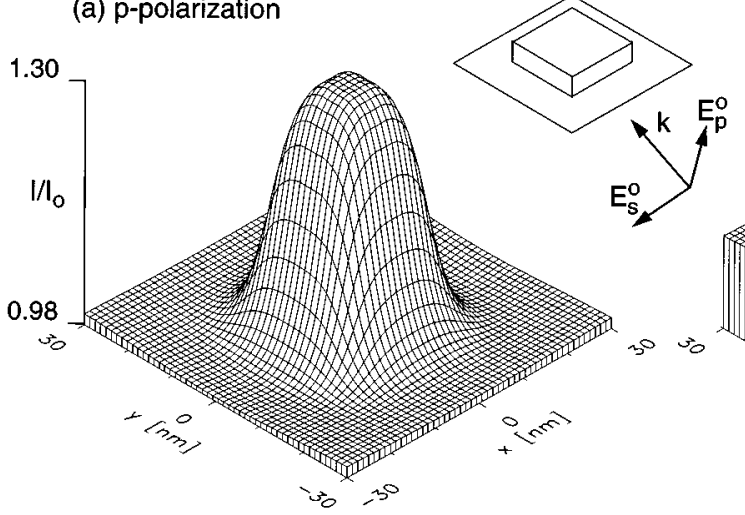

(b) s-polarization

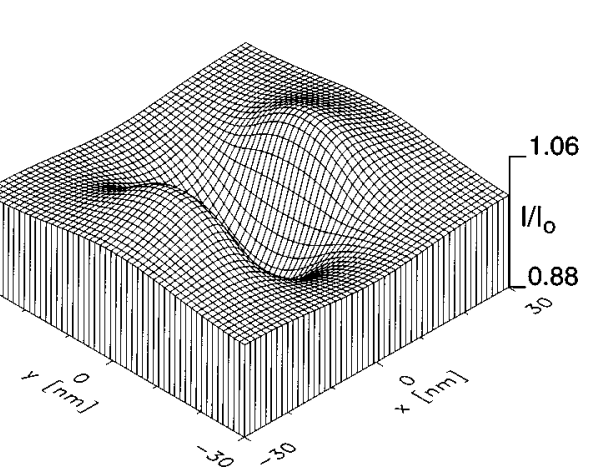

Fig. 2. Same geometry as in Fig. 1 but with another propagation direction for the incident field. 


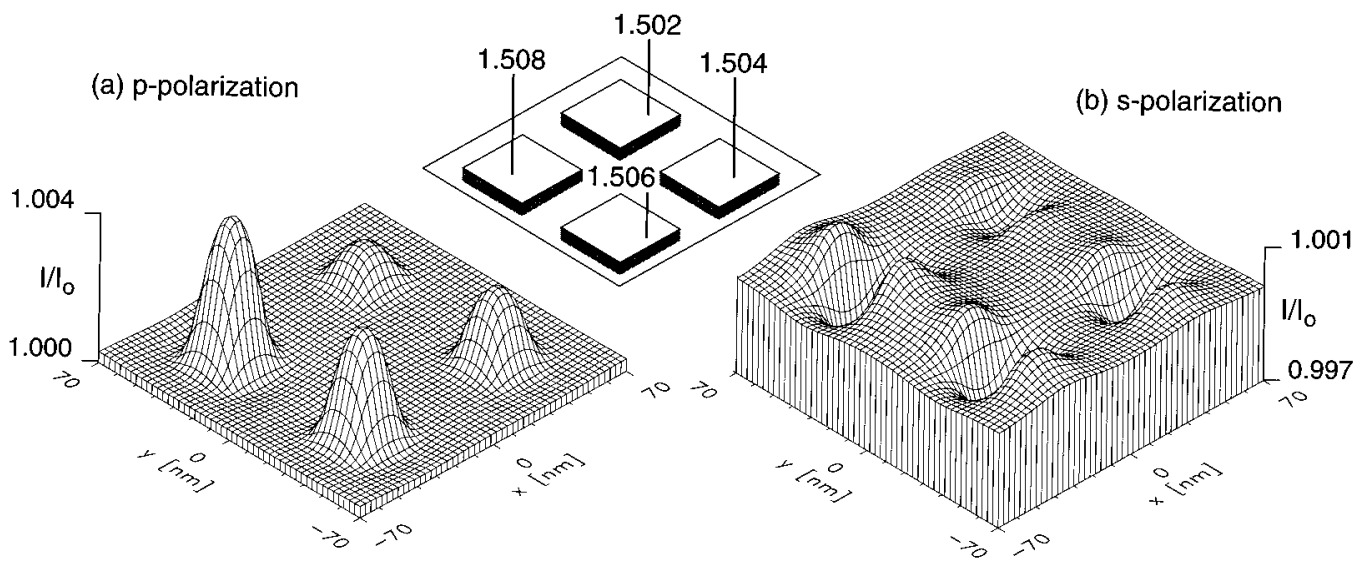

Fig. 3. Relative total field intensity $I / I_{0}, 5 \mathrm{~nm}$ above a perfectly flat surface $(n=1.500)$ with four $20 \times 20 \times 10 \mathrm{~nm}^{3}$ buried pads of different indices (see inset). The total field intensity $I$ is normalized to the value $I_{0}$ measured without buried pads. Same incident fields as in Fig. 1.

these boundary conditions along the protrusion sides parallel to the incident field, and no depolarization effect is observed along these sides. When the orientation of the incident field changes, the depolarization effects appear along other protrusion sides, as is visible in Fig. 2(b).

For $p$ polarization the incident field is mainly vertical, and the dominant depolarization effects occur along the top protrusion face. Because we compute the field in a plane parallel to that top face, we cannot observe these vertical depolarization effects, and we measure only the field enhancement caused by the entire protrusion. Note that, in spite of the small protrusion volume, this enhancement is significant, and the field intensity reaches 1.3 times its value without a protrusion [Fig. 1(a)]. Because this field confinement effect is related to the vertical field component, it does not depend on the propagation direction of the incident field, and the total field intensity always reproduces the surface defect, as can be seen in Figs. 1(a) and 2(a). Nonetheless, let us emphasize that if we considered only the minor horizontal field component that also exists for that $p$ polarization, we would of course observe a horizontal depolarization effect similar to that observed for $s$ polarization; but, for our illumination mode, the total field in $p$ polarization is dominated by the vertical field component, and this horizontal depolarization effect is not visible. For all that, it is important to keep in mind that in a practical SNOM experiment, the polarization sensitivity of the signal-collecting scheme (optical fiber, aperture, etc.) can strongly influence the recorded image. In this way, the experimental image can be dominated by the intensity of a given field component and not reproduce the total near-field intensity.

Let us now turn to a pure dielectric contrast case. We show in Fig. 3 the near-field intensity $5 \mathrm{~nm}$ above a perfectly flat surface with four buried pads with indices slightly different from the surface. The top face of each pad coincides with the surface, so that the system is perfectly flat.

The topography of the field intensity produced by such a buried pad is similar to that obtained for the protrusion

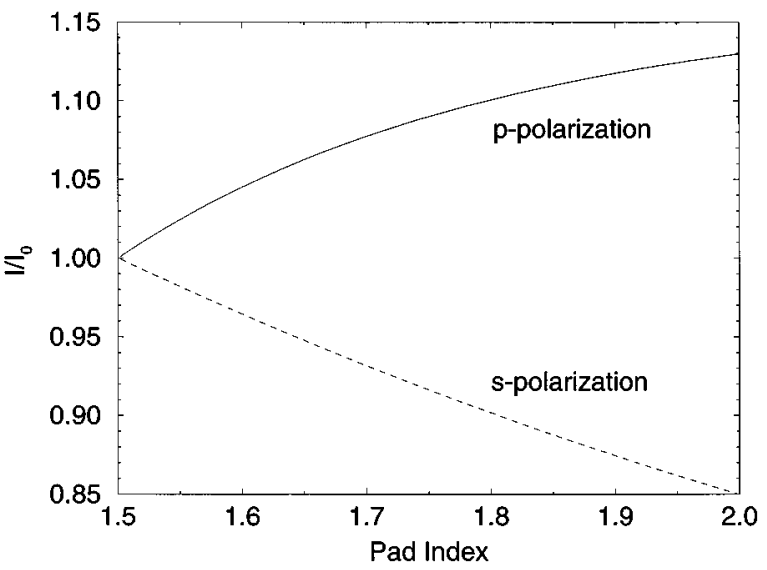

Fig. 4. Relative total field intensity $I / I_{0}, 5 \mathrm{~nm}$ above the center of a $20 \times 20 \times 10 \mathrm{~nm}^{3}$ pad of varying index, buried in a perfectly flat surface $(n=1.500)$. The total field intensity $I$ is computed for the two polarizations depicted in Fig. 1 and normalized to the value $I_{0}$ measured without a buried pad.

on the surface: field confinement above the pad for $p$ polarization [Fig. 3(a)] and inverse contrast and field gradients along the object sides for $s$ polarization [Fig. 3(b)].

In Fig. 3 the amplitude of the signal above a buried pad increases with the contrast of index (difference between pad index $n_{\text {pad }}$ and surface index $n_{\text {surf }}$ ). This effect is emphasized in Fig. 4, where we show the relative total field intensity above the center of a buried pad as a function of the pad index. Note the field enhancement for $p$ polarization and the field depletion for $s$ polarization. For a small contrast index $\left(n_{\text {pad }}=1.5 \ldots 1.6\right)$, the relative total field intensity varies mainly linearly with the pad index (Fig. 4). For a larger contrast, the intensity converges to a limiting value that depends on the pad shape. This effect is similar to the scattering by a sphere of index $n_{\text {sp }}$ and radius $r$ embedded in a homogeneous medium of index $n_{\text {med }}$. For such a system, the scattered field is proportional to the mean polarizability $\alpha$ of the sphere. For 


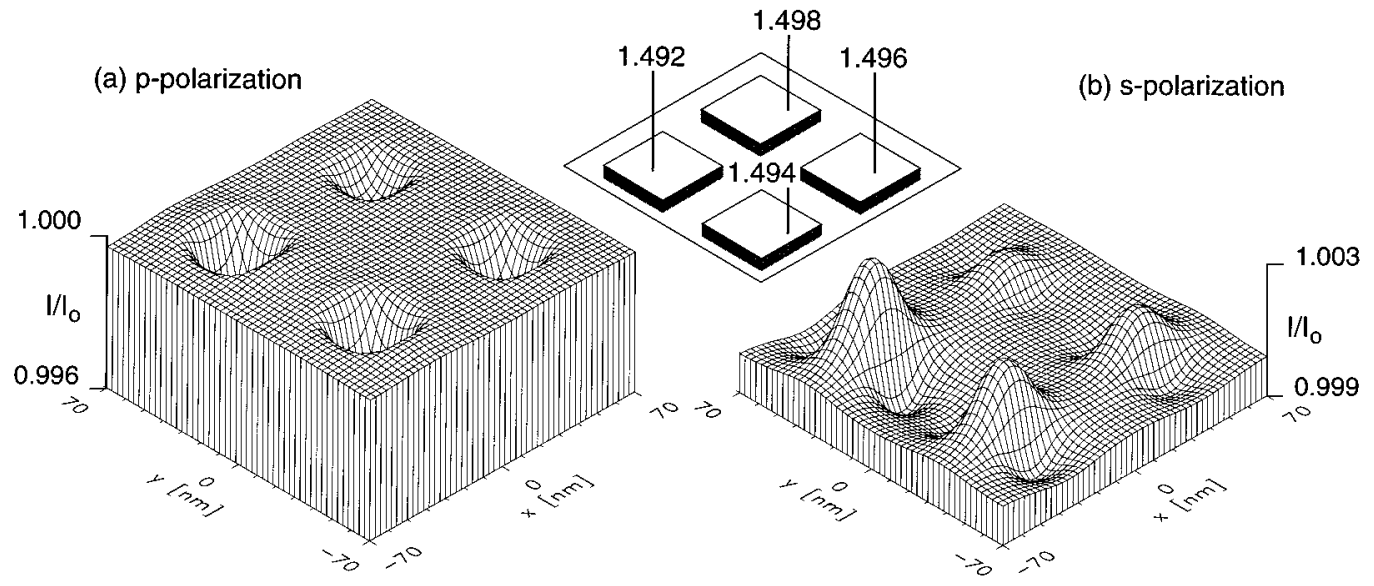

Fig. 5. Same geometry as in Fig. 3 but with a negative index contrast (the indices of the pads are smaller than the surface index). This negative index contrast leads to the reversed (upside down) image of Fig. 3.

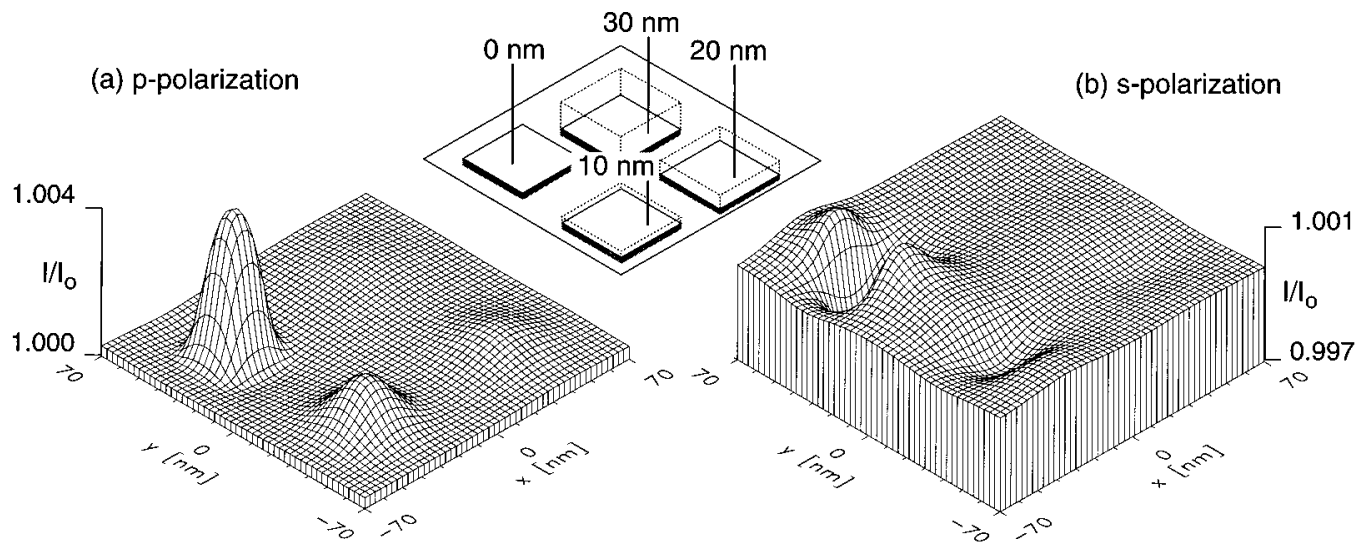

Fig. 6. Relative total field intensity $I / I_{0}, 5 \mathrm{~nm}$ above a perfectly flat surface $(n=1.500)$ with four $20 \times 20 \times 10 \mathrm{~nm}^{3}$ pads $(n=1.508)$ buried at different depths. The distance between the top face of each pad and the substrate-air interface is given in the inset. The total field intensity $I$ is normalized to the value $I_{0}$ measured without buried pads. Same incident fields as in Fig. 1 .

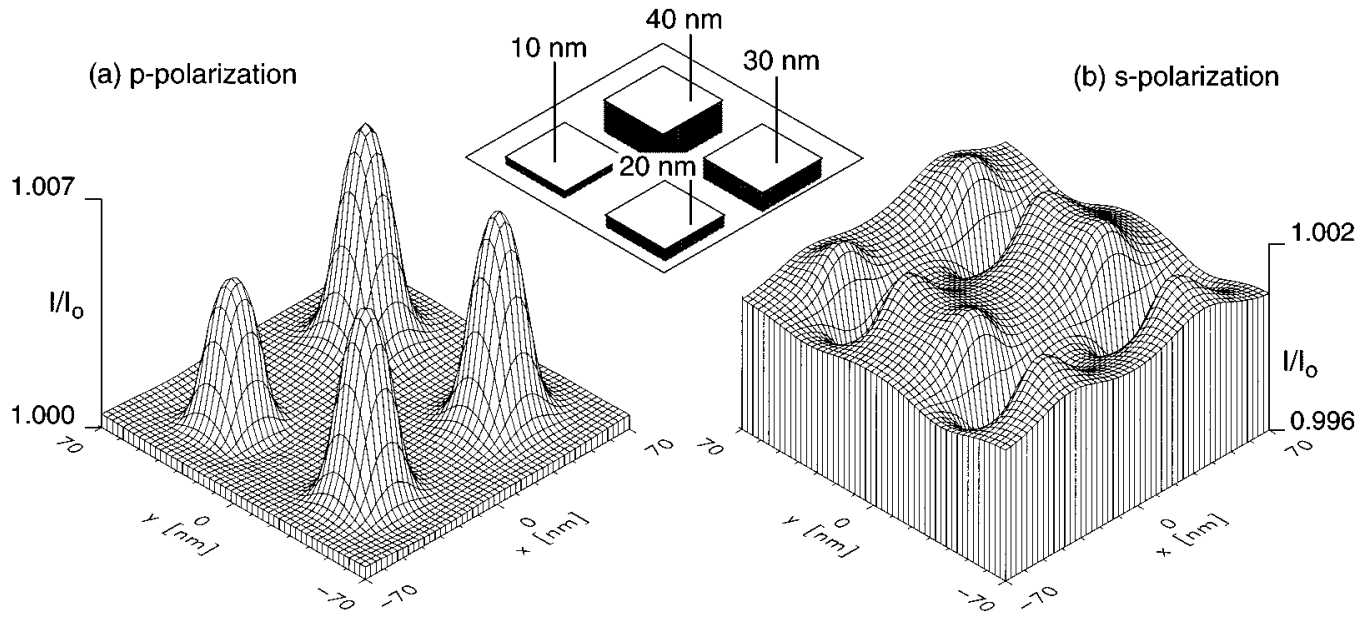

Fig. 7. Relative total field intensity $I / I_{0}, 5 \mathrm{~nm}$ above a perfectly flat surface ( $n=1.500$ ) with four $20 \times 20 \times h \mathrm{~nm}^{3}$ buried pads ( $n=1.508$ ) of varying height: $h=10,20,30$ and $40 \mathrm{~nm}$. The total field intensity $I$ is normalized to the value $I_{0}$ measured without buried pads. Same incident fields as in Fig. 1. 


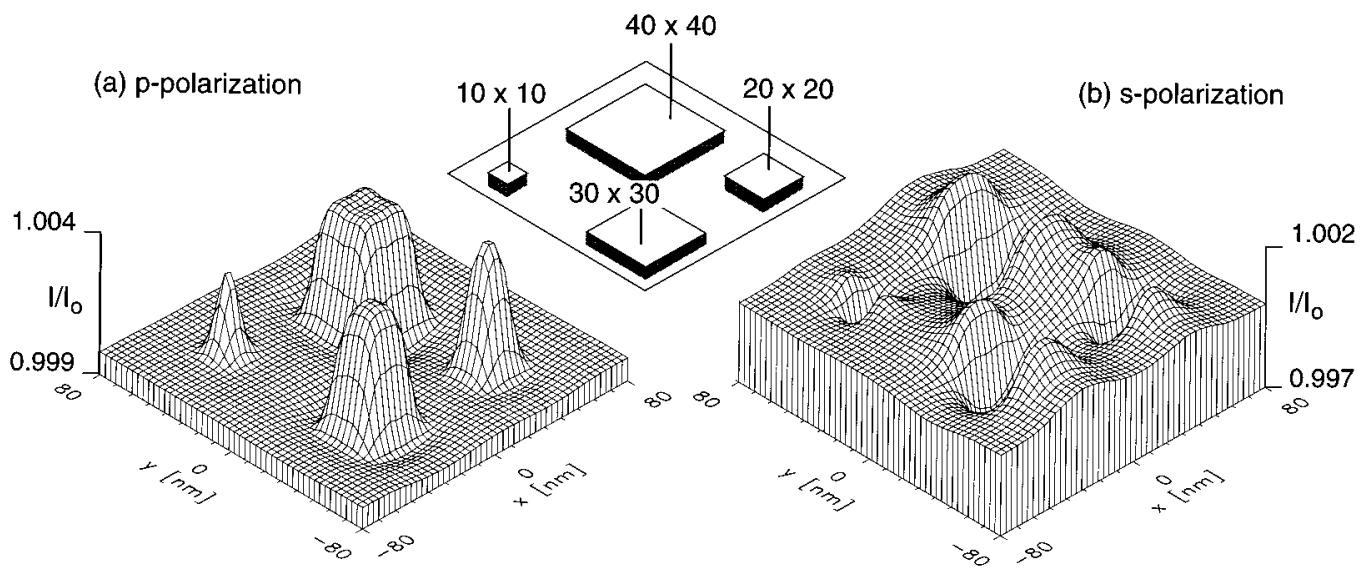

Fig. 8. Relative total field intensity $I / I_{0}, 5 \mathrm{~nm}$ above a perfectly flat surface $(n=1.500)$ with four 10 -nm-thick buried pads $(n=1.508)$ of varying area $\left(10 \times 10,20 \times 20,30 \times 30\right.$, and $\left.40 \times 40 \mathrm{~nm}^{2}\right)$. The total field intensity $I$ is normalized to the value $I_{0}$ measured without buried pads. Same incident fields as in Fig. 1.

(a) p-polarization

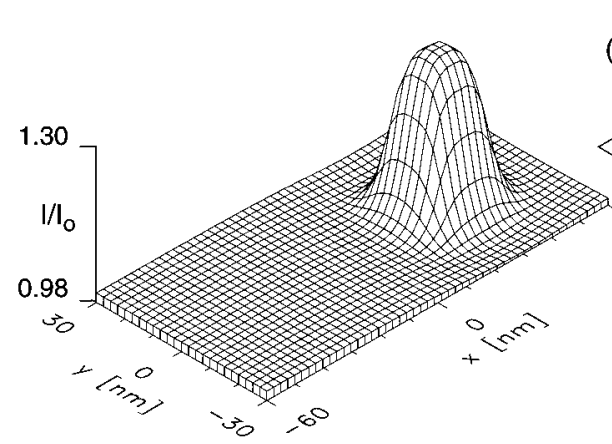

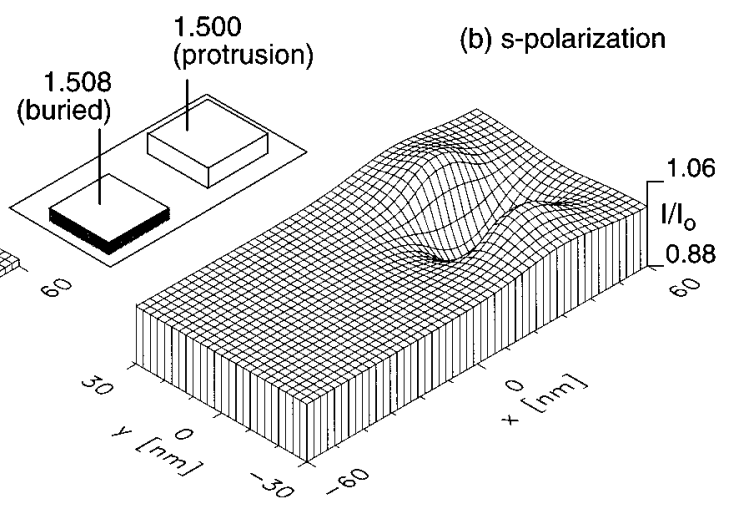

Fig. 9. Relative total field intensity $I / I_{0}$ above a surface $(n=1.500)$ with a $20 \times 20 \times 10 \mathrm{~nm}^{3}$ pad $(n=1.508)$ buried in the surface and a $20 \times 20 \times 10 \mathrm{~nm}^{3}$ protrusion on the surface. The field intensity is computed $5 \mathrm{~nm}$ above the protrusion. The total field intensity $I$ is normalized to the value $I_{0}$ measured on a perfectly flat surface. Same incident fields as in Fig. 1.

a given embedding medium and sphere radius, this mean polarizability, given by the Lorentz-Lorenz formula ${ }^{27}$

$$
\alpha=\frac{n_{\mathrm{sp}}^{2}-n_{\mathrm{med}}^{2}}{n_{\mathrm{sp}}^{2}+2 n_{\mathrm{med}}^{2}} r^{3},
$$

also converges to a constant value $\left(\alpha=r^{3}\right)$ for large sphere indices.

It is important to note that the intensity of the signal measured in Fig. 3 is 100 times smaller than in the topographic contrast case studied in Fig. 1. We will address this problem at the end of this section.

The dielectric contrast investigated in Fig. 3 is positive (the index is higher in the pad than in the surface). If we consider the opposite case, where the pad index is smaller than the surface index, we obtain a reversed field intensity pattern, as is visible in Fig. 5. Note the reversed peak for $p$ polarization and the reversed depletion that leads to a broad peak above each pad for $s$ polarization.

If we place the buried pads not directly below the surface but at a certain depth, we observe a strong decrease of the measured intensity, as can be seen in Fig. 6 . When the pad is only $10 \mathrm{~nm}$ below the surface, the corresponding peak in $p$ polarization reaches only one third of the value measured when the pad was just at the surface [Fig. 6(a)].

This extremely rapid reduction of the field intensity as a function of the pad depth is caused by the strong decay of the evanescent-field components responsible for the near-field intensity. This decay is easily understood if one recalls the form of Green's tensor $\mathbf{G}^{0}$ used for the calculation of the scattered field in our formalism [see, e.g., Eq. (9) of Ref. 23]. Indeed, in addition to the $1 / R$ term that dominates the far field, $\mathbf{G}^{0}$ also contains $1 / R^{2}$ and $1 / R^{3}$ terms that account for the near field and are responsible for the rapid intensity decay observed in Fig. 6 when the pad depth increases ( $R$ can be viewed as the distance between the pad and the point where the field is computed).

When we maintain the pad just below the surface and increase its height, thereby increasing its volume, the measured intensity rises (Fig. 7). However, the intensity is not simply proportional to the pad height, and for very high buried pads the intensity saturates. This is due to 
the two following competing effects: On the one hand, a larger pad volume produces more depolarization signal; but, on the other hand, when the height of the pad increases, its center moves away from the observation point, which decreases the measured field intensity.

If we now keep the pad height constant and vary its area, we observe extremely interesting effects (Fig. 8). For $p$ polarization the intensity reaches a maximum for a $20 \times 20 \mathrm{~nm}^{2}$ pad. For larger pads the enhancement factor is smaller, and the field can even decrease above the center of the pad [Fig. 8(a)]. This effect is similar to that observed in Fig. 2 of Ref. 23. For $s$ polarization this saturation effect does not exist for these dimensions, and the larger the pad area is, the higher are the field gradients along its sides [Fig. 8(b)]. On the other hand, a saturation effect is observed above the center of the pads, and the field intensity there does not continuously decrease as the pad area augments [Fig. 8(b)].

The previous examples illustrate the fact that a topographic and a dielectric object give similar near-field signals with the same dependence on the polarization. Nevertheless, the signal obtained for the topographic object of Figs. 1 and 2 is 100 times larger than the signal obtained for the remaining examples of this paper. Therefore, not only do pure dielectric variations in a surface produce a signal similar to that produced by topographic variations, but the amplitude of this signal becomes insignificant as soon as the surface presents some roughness.

This effect is visible in Fig. 9, where we give the near field above a surface with both a protrusion and a buried pad. For both polarizations the signal of the buried pad is completely screened out by the signal of the protrusion.

This difference of response intensity between two defects that have exactly the same volume $(20 \times 20 \times 10$ $\mathrm{nm}^{3}$ ) originates from the difference of dielectric contrast between the defect and its surrounding. As a matter of fact, the protrusion ( $n=1.500)$ is surrounded by air ( $n=1.000)$, whereas the pad $(n=1.508)$ is buried in a surface with index $n=1.500$.

Therefore, if we increase the index of the buried pad, its signal rises, as is visible in Fig. 10, where we show the near field spawned by a buried pad ( $n=2.000)$ with the same dielectric contrast as the surface protrusion. The fact that the peak associated with the buried pad is still much smaller than the signal provided by the protrusion is caused partially by the observation distance, which is

(a) p-polarization

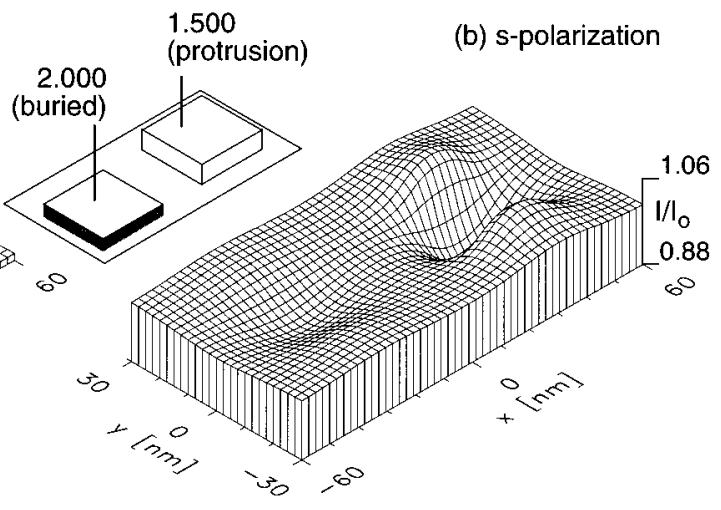

Fig. 10. Same geometry as in Fig. 9, but the index of the buried pad is now $n=2.000$. Therefore the dielectric contrast between the pad and its surrounding is now the same as the contrast between the protrusion and its surrounding (air). The field intensity is computed $5 \mathrm{~nm}$ above the protrusion (i.e., $15 \mathrm{~nm}$ above the buried pad). The total field intensity $I$ is normalized to the value $I_{0}$ measured on a perfectly flat surface. Same incident fields as in Fig. 1.
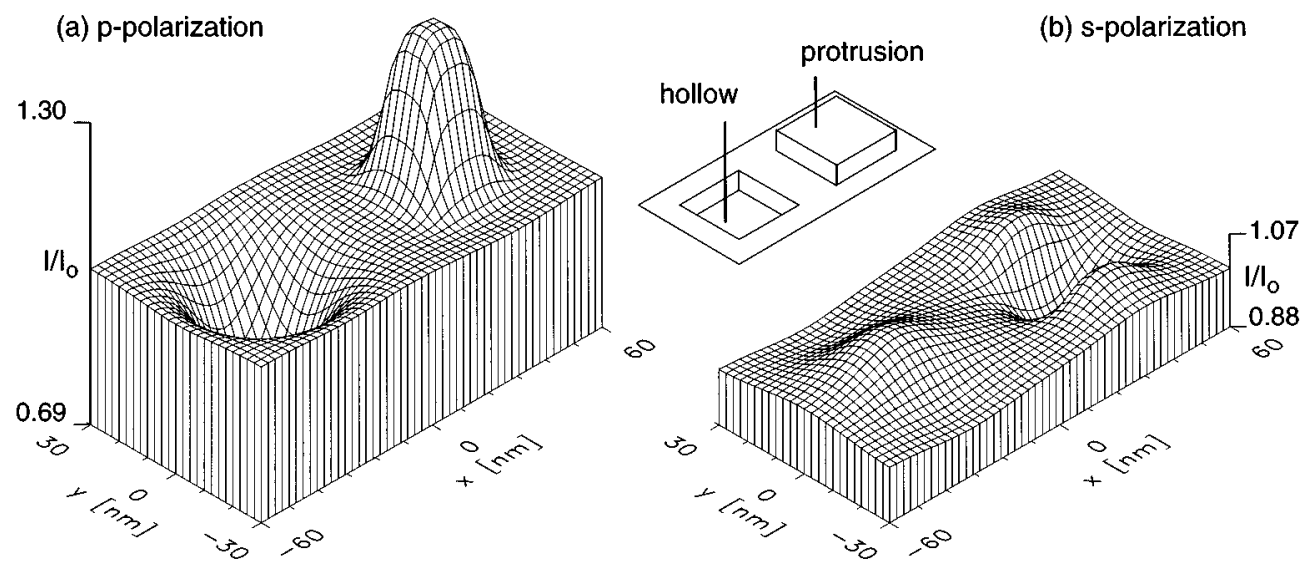

Fig. 11. Relative total field intensity $I / I_{0}$, above a surface $(n=1.500)$ with a $20 \times 20 \times 10 \mathrm{~nm}^{3}$ hollow and a $20 \times 20 \times 10 \mathrm{~nm}^{3}$ protrusion. The field intensity is computed $5 \mathrm{~nm}$ above the protrusion (i.e., $15 \mathrm{~nm}$ above the hollow). The total field intensity $I$ is normalized to the value $I_{0}$ measured on a perfectly flat surface. Same incident fields as in Fig. 1 . 


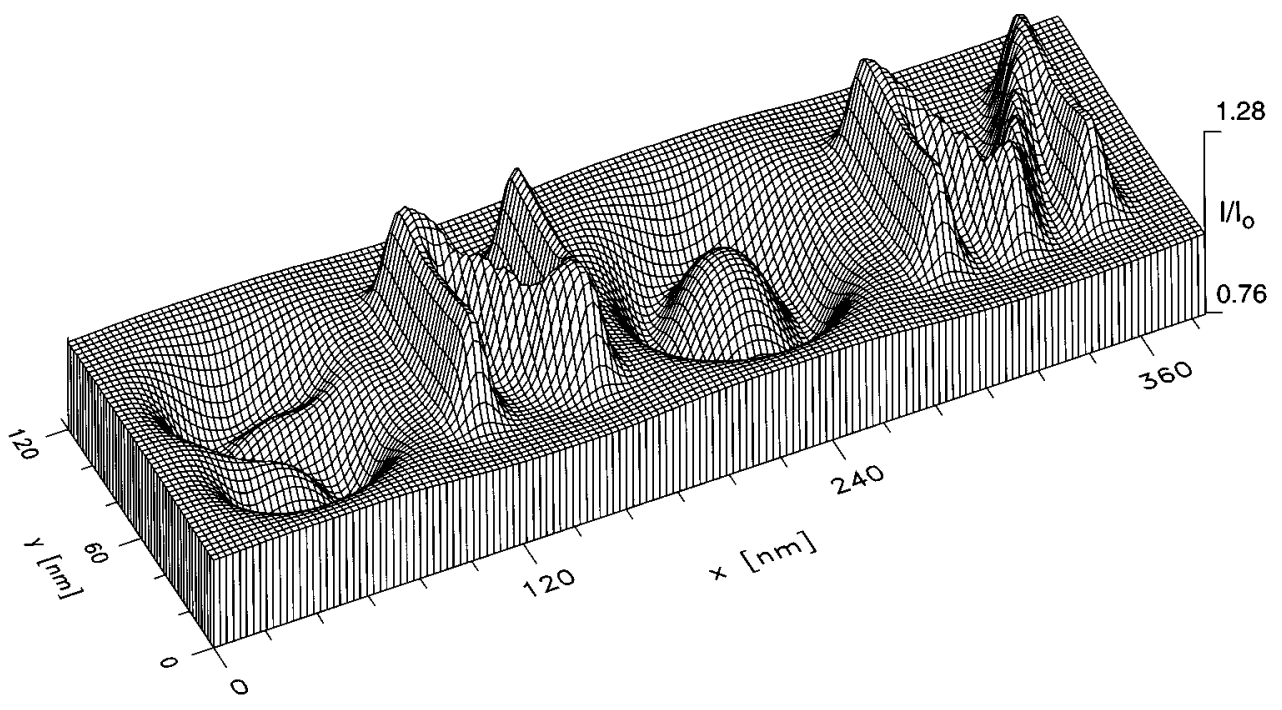

Fig. 12. Relative total field intensity $I / I_{0}, 15 \mathrm{~nm}$ above a dielectric surface $(n=1.500)$ on which the SNOM acronym is reproduced. The letters $\mathrm{S}$ and $\mathrm{O}$ are etched in the surface, whereas the letters $\mathrm{N}$ and $\mathrm{M}$ build on the surface two protrusions of same index. The letters' thickness and linewidth is $10 \mathrm{~nm}$. The total field intensity $I$ is normalized to the value $I_{0}$ measured on a perfectly flat surface. Same incident field as in Fig. 1(a) ( $p$ polarization).

larger for the buried pad $(15 \mathrm{~nm})$ than for the protrusion $(5 \mathrm{~nm})$. However, if we compute the field intensity at the same observation distance $(5 \mathrm{~nm})$ above the buried pad and above the protrusion, we obtain for $p$ polarization a relative field intensity of 1.13 above the pad, whereas it reaches 1.30 above the protrusion. Therefore, although both have the same dielectric contrast with their respective surrounding, the buried pad produces a much smaller field enhancement than the protrusion. This is due to the important screening that takes place in the substrate at the pad interfaces. This screening reduces the field inside the buried pad, thereby decreasing the near-field signal. Such an effect does not exist for the protrusion, because the latter has the same index as the surface.

As a final example, we give in Fig. 11 the near field spawned above a surface with both a protrusion and a hollow. This hollow can be viewed as a buried pad with an index $n=1.00$, i.e., with a negative dielectric contrast to the surface. The difference of dielectric contrast between the protrusion and the hollow leads to opposite near-field intensity patterns (Fig. 11).

\section{CONCLUSION}

We have shown that dielectric contrast (i.e., 3D subwavelength index variations buried in a perfectly flat surface) gives a near-field response similar to that of topographic contrast (e.g., protrusion on the surface).

The relationship between the signal produced by a buried object and its index is rather subtle. It depends strongly on the dielectric contrast between the object and its surrounding material. Therefore a buried pad with an index similar to that used to define buried waveguides in PIC's produces such a small signal that it can disappear into the background caused by surface roughness. When the dielectric contrast increases, the signal in- creases in intensity. But for larger contrasts, a saturation of the signal intensity is observed.

The object area has a limited influence on the field intensity, and for a large-area subwavelength defect, the near field in $p$ polarization reaches a maximum and remains flat. On the other hand, an extremely important parameter is the object depth. Indeed, when the defect is not located directly below the surface, its signal intensity decreases extremely rapidly. For a negative dielectric contrast, i.e., when the buried object has a smaller index than the substrate, the total field intensity pattern is reversed. In particular, a hollow in the surface produces the reversed near-field intensity as a protrusion on the surface.

This effect is illustrated in Fig. 12, where we give the near-field intensity spawned above a surface on which the SNOM acronym is reproduced. The letters $\mathrm{S}$ and $\mathrm{O}$ are etched in the surface, whereas the letters $\mathrm{N}$ and $\mathrm{M}$ build two protrusions on the surface. The letters' thickness and linewidth is $10 \mathrm{~nm}$. This figure also illustrates the power and versatility of the generalized field propagator technique for the investigation of arbitrary geometries. ${ }^{23}$

\section{ACKNOWLEDGMENTS}

It is a pleasure to acknowledge stimulating discussions with G. L. Bona, D. Courjon, L. Novotny, and D. W. Pohl. Olivier J. F. Martin gratefully acknowledges the support of the Swiss National Science Foundation through Priority Programme NFP36.

Olivier J. F. Martin's e-mail address is martin @ifh.ee.ethz.ch

\section{REFERENCES}

1. For a recent overview of SNOM, see D. W. Pohl and D. 
Courjon, eds., Near Field Optics, NATO ASI Series E (Kluwer, Dordrecht, 1993) and also the special issue of Ultramicroscopy 57(2/3) (February 1995).

2. F. M. Depasse and D. A. Courjon, "Modeling of the field transfer through thick dielectric lines: use in linewidth measurement," Appl. Optics 30, 1355-1360 (1991).

3. C. Girard and A. Dereux, "Optical spectroscopy of a surface at the nanometer scale: a theoretical study," Phys. Rev. B 49, 11344-11351 (1994).

4. F. Pincemin, A. Sentenac, and J.-J. Greffet, "Near field scattered by a dielectric rod below a metallic surface," J. Opt. Soc. Am. A 11, 1117-1127 (1994).

5. C. Girard, A. Dereux, O. J. F. Martin, and M. Devel, "Importance of confined fields in near-field optical imaging of subwavelength objects," Phys. Rev. B 50, 14467-14473 (1994).

6. L. Novotny, D. W. Pohl, and P. Regli, "Light propagation through nanometer-sized structures: the two-dimensionalaperture scanning near-field optical microscope," J. Opt. Soc. Am. A 11, 1768-1779 (1994).

7. R. Carminati, A. Madrazo, and M. Nieto-Vesperinas, "Electromagnetic wave scattering from a cylinder in front of a conducting surface-relief grating," Opt. Commun. 111, 26-33 (1994).

8. L. Novotny, D. W. Pohl, and B. Hecht, "Light confinement in scanning near-field optical microscopy," Ultramicroscopy (to be published).

9. C. Girard, A. Dereux, and O. J. F. Martin, "Theory of nearfield optics," in Photon and Local Probes, O. Marti and R. Möller, eds., NATO ASI Series E (Kluwer, Dordrecht, 1995), pp. 1-20.

10. N. García and M. Nieto-Vesperinas, "Near-field optics inverse scattering reconstruction of reflective surfaces," Opt. Lett. 18, 2090-2092 (1993).

11. N. García and M. Nieto-Vesperinas, "Rough surface retrieval from the specular intensity of multiply scattered waves," Phys. Rev. Lett. 71, 3645-3648 (1993).

12. J.-J. Greffet, A. Sentenac, and R. Carminati, "Surface profile reconstruction using near-field data," Opt. Commun. 116, 20-24 (1995)

13. N. García and M. Nieto-Vesperinas, "Direct solution to the inverse scattering problem for surfaces from near-field intensities without phase retrieval," Opt. Lett. 20, 949-951 (1995).

14. F. Pincemin, A. Sentenac, and J.-J. Greffet, "Near field scattered by subsurface particles," in Near Field Optics, D.
W. Pohl and D. Courjon, eds., NATO ASI Series E (Kluwer, Dordrecht, 1993), pp. 209-220.

15. D. Barchiesi and D. Van Labeke, "PSTM: an alternative to measure local variations of optical index," Microsc. Microanal. Microstruct. 5, 435-446 (1994).

16. R. Carminati and J.-J. Greffet, "Influence of dielectric contrast and topography on the near field scattered by an inhomogeneous surface," J. Opt. Soc. Am. A 12, 2716-2725 (1995).

17. M. Kawachi, "Silica waveguides on silicon and their application to integrated-optic components," Opt. Quantum Electron. 22, 391-416 (1990).

18. C. H. Henri, G. E. Blonder, and R. F. Kazarinov, "Glass waveguides on silicon for hybrid optical packaging," J. Lightwave Technol. 7, 1530-1539 (1989).

19. N. H. G. Baken, M. B. J. Diemeer, J. M. Van Splunter, and H. Blok, "Computational modeling of diffused channel waveguides using a domain integral equation," J. Lightwave Technol. 8, 576-586 (1990).

20. A. Goo Choo, H. E. Jackson, U. Thiel, G. N. De Brabander, and J. T. Boyd, "Near field measurements of optical channel waveguides and directional couplers," Appl. Phys. Lett. 65, 947-949 (1994).

21. N. van Hulst, M. Moers, and E. Borgonjen, "Applications of near field optical microscopy," in Photon and Local Probes, O. Marti and R. Möller, eds., NATO ASI Series E (Kluwer, Dordrecht, 1995), pp. 165-180.

22. E. Bourillot, F. De Fornel, J. P. Goudonnet, D. Persegol, A. Kevorkian, and D. Delacourt, "Analysis of photon-scanning tunneling microscope images of inhomogeneous samples: determination of the local refractive index of channel waveguides," J. Opt. Soc. Am. A 12, 95-106 (1995).

23. O. J. F. Martin, C. Girard, and A. Dereux, "Generalized field propagator for electromagnetic scattering and light confinement," Phys. Rev. Lett. 74, 526-529 (1995).

24. O. J. F. Martin, A. Dereux, and C. Girard, "Iterative scheme for computing exactly the total field propagating in dielectric structures of arbitrary shape," J. Opt. Soc. Am. A 11, 1073-1080 (1994)

25. D. Courjon, C. Bainier, and M. Spajer, "Imaging of submicron index variations by scanning optical tunneling," J. Vac. Sci. Technol. B 10, 2436-2439 (1992).

26. C. Kittel, Introduction to Solid State Physics, 6th ed (Wiley, New York, 1986).

27. M. Born and E. Wolf, Principles of Optics, 6th ed. (Pergamon, Oxford, 1980). 